\title{
FORECASTING OF THE EMERGENCE AND DEVELOPMENT OF INNOVATIVE TECHNOLOGIES
}

\author{
Andrey V. Proletarsky, Ark M. Andreev, Dmitry V. Berezkin, \\ Ilya A. Kozlov and Moudar Kiwan \\ Bauman Moscow State Technical University, Moscow, Russia
}

\begin{abstract}
Under "Industry 4.0" conditions, the automatic prediction of emergence and evolution of innovative technologies is an important challenge. To solve this problem, a hybrid approach to big data streams analysis is proposed and developed. This approach allows the automated monitoring and forecasting of situations development through processing streams of heterogeneous data which is presented, in particular, by text documents, time series and database records. The proposed approach includes detecting events in data streams, forming situations, identifying possible scenarios for their further development and preparing suggestions for decision makers. To represent events that are reflected in a stream of structured data, a frame-based model is proposed. To form situational chains that reflect the development of innovative technologies, an incremental clustering method is used. Forecasting further evolution of the analyzed innovative technology consists in forming possible scenarios for the situation's development based on the case-based approach. An example of an event and a situation detected in a stream of structured data, as well as an example of a scenario generated for the detected situation are presented.
\end{abstract}

\section{KEYWORDS}

Industry 4.0, Situational Analysis, Forecasting, Decision Support System, Scenario Analysis, Clustering

\section{INTRODUCTION}

The development of modern society is constantly accelerating, and the changes occurring in it affect political, economic, social and other areas of public life (Toffler, 1970). A great part of these changes are associated with the emergence, development and synthesis of technologies in physical, digital and biological fields (Schwab, 2016). The human environment is gradually turning into a cyber-physical system, which leads to a significant change in social and economic relations. This phenomenon is described in the works of modern researchers by such terms as "The Fourth Industrial Revolution", "Digital Economy", and "Industry 4.0".

Since changes taking place in society are inseparably associated with new technologies, many categories of specialists (scientists, engineers, economists, politicians, sociologists, ecologists, etc.) need to predict their emergence and development, as well as identify possible consequences and potential threats caused by their spreading. These forecasts are necessary to make the best management decisions regarding the creation, development, implementation and usage of new industry 4.0 technologies.

Existing approaches to this task usually rely on expert analysis (Choi and Choi, 2015). They require a great amount of efforts and time and can only answer some questions regarding the strategic development of science and technology for certain areas of the country's economy. In this regard, developing an automated forecasting method is a highly relevant task. The method should require minimal participation of experts and be easily adaptable for analyzing various areas. This article is dedicated to the development of such a method. 


\section{THE CHOICE OF APPROACH TO FORECASTING THE DEVELOPMENT OF TECHNOLOGIES}

One of the main features of the "Fourth Industrial Revolution" is the possibility and necessity of leveraging big data streams to solve various analytical tasks. Thus, predicting the emergence and development of new technologies can be performed based on analyzing huge volumes of data dynamically coming from open and specialized sources such as news portals, corporate databases, and third-party information systems. The dynamic nature of data streams allows tracing the history of a technology's development over time, and this history should be taken into account when forecasting its future development.

The main properties of big data are usually called "three Vs": volume, velocity and variety. The data has a huge volume and velocity, which imposes restrictions on processing methods: one has to use methods that are able to amplify the analysis results when new data is received without performing a full recalculation based on all the information collected. In addition, big data is varied, and therefore the processing methods should be able to work with various types of structured and unstructured information.

Among the existing forecasting methods, an important place is occupied by time series forecasting, which implies that the value of a certain function at the first $(n)$ points of the time series is known. Using this information, it is necessary to predict the value at the $(n+1)$ point of the time series. A wide range of methods is used to predict time series: regression models, autoregressive models (ARIMA, GARCH), exponential smoothing model, moving average model and others (Shumway and Stoffer, 2017).

In many practical tasks, the data is represented not by time series, but by vectors of attributes: $x_{i}=\left(x_{i}^{1}, x_{i}^{2}, \ldots, x_{i}^{n}\right)$. In this case, forecasting can be considered as a classification task (Konnova and Basarab, 2018). Approaches based on classification imply assigning the analyzed object to one of several classes: $y \in\left\{y^{1}, y^{2}, \ldots, y^{N}\right\}$. Efficient methods such as decision trees, the nearest neighbor method, a naive Bayes classifier, logistic regression, support vector machines, and others are widely used to build classification models (Hastie, Tibshirani and Friedman, 2009).

These methods make forecasts automatically and take the dynamics of the situation into account. However, they are designed to analyze specific types of data and cannot be applied to heterogeneous data streams. Furthermore, the forecasting results that they yield cannot be directly used to prepare recommendations for decision makers (DM): the generation of such proposals is a separate task.

In order to eliminate these shortcomings, it was decided to use a hybrid approach to the heterogeneous data streams analysis (Andreev, Berezkin and Kozlov, 2018) to predict the development of new technologies. The approach consists of the following steps:

1. Detecting events $\varepsilon_{i}$ related to a certain technology;

2. Forming a chain of interrelated events which reflects the development of the technology over time. Such a chain will be called a situation $s=\left(\varepsilon_{s}^{1}, \varepsilon_{s}^{2}, \ldots, \varepsilon_{s}^{n}\right)$;

3. Determining possible variants of continuation of the formed situational chain. These variants will be called scenarios $\xi=\left(\varepsilon_{\xi}^{1}, \varepsilon_{\xi}^{2}, \ldots, \varepsilon_{\xi}^{m}\right)$;

4. Determining the pessimistic, optimistic and most probable scenarios;

5. Preparing recommendations for the DM regarding actions that should be taken to promote the development of the situation according to the most profitable scenario.

\section{DETECTING EVENTS IN DATA STREAMS}

Detecting events and tracking situations is performed via various methods depending on the type of data (Deviatkov and Lychkov, 2017; Lychkov, Alfimtsev and Sakulin, 2018). To detect events in a stream of text documents, the authors proposed a method based on incremental clustering (Andreev, Berezkin and Kozlov, 2017).

When processing a stream of structured data (such as records of corporate databases), each event corresponds to a certain record in a database containing data about the companies' activities regarding creating, developing and implementing innovating technologies. In this regard, a convenient way to represent an event is a frame with slots describing properties of the event: $\varepsilon_{i}=\left(\left(a_{i}^{1}, v_{i}^{1}\right),\left(a_{i}^{2}, v_{i}^{2}\right), \ldots,\left(a_{i}^{f}, v_{i}^{f}\right)\right)$, where 
$a_{i}^{k}$ and $v_{i}^{k}$ are the name and the value of the $k$-th slot of the $i$-th event and $f$ is the number of slots. When using the frame representation event detection consists in filling the slots of the frame with data extracted from the corresponding fields of the record. Table 1 shows an example of a frame representation of an event that is an acquisition of Trustev, a company developing solutions for online verification, by TransUnion. As shown in the table, the slots contain data of different types - numeric values, dates, named entities, text.

Table 1. Example of a frame representation of an event

\begin{tabular}{ll}
\hline Slot Name & Slot Value \\
\hline Acquiring company & TransUnion \\
Target company & Trustev \\
Deal date & 09.12 .2015 \\
Deal sum & $\$ 44$ million \\
Deal description & Trustev Limited, a provider of digital online verification \\
& technology, has been acquired by TransUnion \\
\hline
\end{tabular}

Formation of situational chains based on a set of events can be considered as a clustering task: all events should be divided into groups $C=\left\{c_{i}\right\}$, each of which corresponds to a certain technology. Then events of each group $c_{i}$ can be used to construct a chain $s_{i}$ by ordering the events by the time of occurrence. There are various algorithms that divide multiple objects into clusters (Xu and Tian, 2015): graph, hierarchical, statistical (EM-algorithm and k-means), density-based (DBSCAN). However, all of these methods perform static clustering, which involves analyzing the entire set of events each time it changes. When processing a stream of structured data, new events constantly occur, so it is more convenient to construct situations by applying dynamic clustering, which allows processing each new event as it appears.

Table 2 demonstrates an example of a situation formed based on structured data stream analysis. The presented situation reflects the development of the technology of online verification of user identity.

Table 2. An example of a situation detected in a stream of structured data

\begin{tabular}{ll}
\hline Date & Event description \\
\hline 11.03 .2014 & Trulioo receives \$6 million in investments \\
26.06 .2014 & Black Score receives initial funding of \$2 million. \\
31.07 .2014 & Confident Technologies receives \$5.59 million of investments \\
09.12 .2015 & TransUnion acquires Trustev \\
\hline
\end{tabular}

\section{GENERATING SCENARIOS}

After building a chain of events that reflects the development of the innovative technology over time, it is necessary to identify possible scenarios for its further development. Scenario generation is based on the case-based approach (Kozaev et al., 2017): the current situation $s_{c}$ is compared to the sample situations $s_{e} \in S_{e}$ where $S_{e}$ is a base of samples prepared by experts. Such samples reflect the development of various technologies in the past. If the current situation is similar to the initial part $s t\left(s_{e}, s_{c}\right)$ of the sample chain, it can be assumed that further development of $s_{c}$ will be similar to the final part $\operatorname{fin}\left(s_{e}, s_{c}\right)$ of the sample chain.

It is assumed that situations can be similar only if there is a pairwise proximity between some of their events. Therefore, comparing situational chains starts with detecting pairs of similar events in these chains. To determine the similarity of the events $\varepsilon_{i}$ and $\varepsilon_{j}$, the distance between them $\gamma_{a n}\left(\varepsilon_{i}, \varepsilon_{j}\right)$ is calculated. If the distance is less than the threshold value $T h_{a n}$, it is concluded that $\varepsilon_{i}$ is analogous to $\varepsilon_{j}$. When calculating the distance $\gamma_{a n}\left(\varepsilon_{i}, \varepsilon_{j}\right)$, the similarity between events is estimated in terms of various criteria, and the set of criteria depends on the domain. The distance is determined as the weighted sum $\gamma_{a n}\left(\varepsilon_{i}, \varepsilon_{j}\right)=\sum_{k=1}^{R} \gamma_{i, j}^{k} \lambda^{k}$, where $R$ is the number of criteria taken into account, $\gamma_{i, j}^{k}$ is the distance between the $i$-th and $j$-th event in terms of the $k$-th criterion, $\lambda^{k}$ is the coefficient that reflects the importance of the $k$-th criterion. The values of coefficients $\lambda^{k}$ and the threshold value $T h_{a n}$ are calculated via the Support Vector Machine method.

The distance between events in terms of a certain criterion is determined by comparing the corresponding components of their models. When processing homogeneous data, the models of all events are generated 
uniformly and consist of the same components. When processing data streams of different types, event models are constructed in different ways, but these models can contain similar components. As an example, an event "Acquisition of an innovative startup by a technological giant" can be considered. When working with structured data, such components of the event model as "Deal participants" and "Deal sum" can be obtained by extracting the values of certain fields of a database record. When analyzing a text stream, these components can also be constructed, but it requires extracting named entities and numerical values from the documents' texts. The presence of similar components in the models allows to determine the similarity between the events in terms of certain criteria and, therefore, to calculate the distance $\gamma_{a n}\left(\varepsilon_{i}, \varepsilon_{j}\right)$ between them. Thus, the similarity between events detected in data streams of different types can be determined, which allows the proposed approach to be used for simultaneous analysis of heterogeneous data streams.

After detecting pairs of similar events in the chains, the similarity between the current situation $s_{c}$ and the sample chain $s_{e}$ is determined. The probability $\mathrm{P}_{\mathrm{an}}\left(s_{e}, s_{c}\right)$ that $s_{c}$ is analogous to $s_{e}$ is calculated via a method based on logistic regression (Andreev, Berezkin and Kozlov, 2018). If $\mathrm{P}_{\mathrm{an}}\left(s_{e}, s_{c}\right)>0.5$, the final part fin $\left(s_{e}, s_{c}\right)$ of $s_{e}$ is considered as a possible scenario for the further development of the current situation.

\section{RESULTS AND DISCUSSION}

Using the proposed method, scenarios were generated for the situation that reflects the development of the technology of online verification of user identity. The most probable scenario and the corresponding suggestions for the decision maker are presented in Table 3.

Table 3. An example of the most probable scenario and recommendations generated for a situation

\begin{tabular}{ll}
\hline Scenario for the current situation's development & $\begin{array}{l}\text { Further development of the technology and receiving new } \\
\text { patents } \\
\text { Development of the reputation-based VoIP connection } \\
\text { management technology } \\
\begin{array}{l}\text { Sample situation that is analogous to the current } \\
\text { one }\end{array}\end{array}$ \\
$\begin{array}{l}\text { The expected period of the scenario's } \\
\text { implementation }\end{array}$ & $\begin{array}{l}\text { Direct funds to the development of the technology within } 6 \\
\text { months }\end{array}$ \\
\hline
\end{tabular}

Also it is necessary to provide the DM with information about differences between the current chain and the sample situation that was used as a basis for the scenario. Even if the situations are considered analogous, they have certain structural and substantive differences that should be taken into account when utilizing the received forecasting results. For this purpose the DM is provided with the following information:

- The most important events of each situation that have no analogues in the other situation.

- The most distinctive words and sentences extracted from textual descriptions of the current and sample situation. These words and sentences describe the most significant features of each situation.

As a way of the further development of the approach, it is proposed to take into account interests and actions of other participants while generating scenarios for the situation. For this purpose, game theory methods can be used. Also more effective use of the forecasting results may be achieved if they are presented to the DM using methods of cognitive graphics, in particular, the method of dynamic meta-anamorphosis (Terekhov et al., 2019). Applying these methods to the problem of innovative technologies development forecasting is a subject of further research.

\section{CONCLUSION}

The article describes the use of a hybrid approach of situational analysis to forecast the emergence and development of innovative technologies. Forecasting is based on processing big streams of data that can be presented in textual, numeric and tabular form. The proposed approach is based on the sequential detection of events in data streams, formation of situations and generation of scenarios for their further development. 
When analyzing streams of structured data, events are represented by frames. Their slots are filled with data extracted from database records. Events are combined into chains (situations) reflecting the development of technologies over time via incremental clustering. After the formation of a situational chain, possible scenarios for its further development are generated. Scenario generation is based on the case-based approach: the current situation is compared to the samples from the database prepared by experts. Samples recognized as analogues of the current situation, are considered as scenarios for its further development.

To test the proposed approach, a system for automated monitoring and forecasting of situations development was developed. Experiments were conducted that proved the system to be able to predict the emergence and evolution of new Industry 4.0 technologies based on the analysis of big data streams.

\section{REFERENCES}

Andreev, A.M., Berezkin, D.V. and Kozlov, I.A., 2017. Podkhod k avtomatizirovannomu monitoringu tem na osnove obnaruzheniya sobytiy v potoke tekstovykh dokumentov [Automated topic monitoring based on event detection in text stream]. Informatsionno-izmeritel'nye i upravlyayushchie sistemy [Information-measuring and Control Systems], Vol. 15, No. 3, pp. 49-60 (in Russ.).

Andreev, A.M., Berezkin, D.V. and Kozlov, I.A., 2018. 'Approach to Forecasting the Development of Situations Based on Event Detection in Heterogeneous Data Streams' in Kalinichenko, L., Manolopoulos, Y., Malkov, O., Skvortsov, N., Stupnikov, S., Sukhomlin, V. (eds.) Data Analytics and Management in Data Intensive Domains. DAMDID/RCDL 2017. Communications in Computer and Information Science. Springer, Cham, Switzerland, Vol. 822, pp. 213-229.

Choi, M. and Choi, H., 2015. Foresight for Science and Technology Priority Setting in Korea. Foresight and STI Governance, Vol. 9, No. 3, pp. 54-67.

Deviatkov, V.V. and Lychkov, I.I., 2017. Recognition of dynamical situations on the basis of fuzzy finite state machines. Proceedings of the International Conferences on Computer Graphics, Visualization, Computer Vision and Image Processing 2017 and Big Data Analytics, Data Mining and Computational Intelligence 2017. Moscow, Russia, pp. 103-109.

Hastie, T., Tibshirani, R. and Friedman, J., 2009. The elements of statistical learning, 2nd edition. Springer-Verlag, New York, USA.

Kozaev, A.T. et al., 2017. Application of case-based method to choose scenarios to resolve emergency situations on main gas pipeline. Proceedings of the 5th International Conference on Actual Problems of System and Software Engineering Supported by Russian Foundation for Basic Research. Moscow, Russia, pp. 120-126.

Konnova, N.S. and Basarab, M.A., 2018. Identification of Functional States of the Cardiovascular System According to Flowmetry Data Using Machine Learning Methods. 11th International Symposium on Computational Intelligence and Design (ISCID). Hangzhou, China, Vol. 1, pp. 221-224.

Lychkov, I.I., Alfimtsev, A.N. and Sakulin, S.A., 2018. Tracking of Moving Objects with Regeneration of Object Feature Points. Proceedings of the 2018 Global Smart Industry Conference (GloSIC). Chelyabinsk, Russia, pp. 1-6.

Schwab, K., 2016. The Fourth Industrial Revolution. Crown Publishing Group, New York, USA.

Shumway, R.H. and Stoffer, D.S., 2017. Time series analysis and its applications: with R examples, 4th edition. Springer, Cham, Switzerland.

Terekhov, V.I. et al., 2019. Cognitive Visualization in Management Decision Support Problems. Optical Memory and Neural Networks, Vol. 28, No. 1, pp. 27-35.

Toffler, A., 1970. Future Shock. Random House, New York, USA.

Xu, D. and Tian, Y., 2015. A comprehensive survey of clustering algorithms. Annals of Data Science, Vol. 2, No. 2, pp. 165-193. 